\title{
Long-Term Dependence in Exchange Rates
}

\author{
A. KARYTINOS ${ }^{a}, *$ A.S. ANDREOU ${ }^{b}$ and G. PAVLIDES ${ }^{b}$ \\ ${ }^{a}$ Warwick Business School, University of Warwick, Coventry, CV47AL, UK, \\ ${ }^{\mathrm{b}}$ Department of Computer Engineering \& Informatics, University of Patras, Patras 26500, Greece
}

(Received 4 February 1999)

\begin{abstract}
The extent to which exchange rates of four major currencies against the Greek Drachma exhibit long-term dependence is investigated using a $R / S$ analysis testing framework. We show that both classic $R / S$ analysis and the modified $R / S$ statistic if enhanced by bootstrapping techniques can be proven very reliable tools to this end. Our findings support persistence and long-term dependence with non-periodic cycles for the Deutsche Mark and the French Franc series. In addition a noisy chaos explanation is favored over fractional Brownian motion. On the contrary, the US Dollar and British Pound were found to exhibit a much more random behavior and lack of any long-term structure.
\end{abstract}

Keywords: $R / S$ analysis, V-statistics, Bootstrapping, Persistency, Noisy chaos

JEL Classification: C14, C15, C22, F31

\section{INTRODUCTION}

The long-term behavior of discrete time dynamical systems in Economics and Finance has increased much attention in the recent literature. In this framework extensive mathematical research over the last few years has focused on important issues such as stock exchange and foreign currency exchange rates. In the latter case especially, where a pure trading market is involved, time evolution of data is more inclined to follow fads and fashions. This type of behavior follows from the fact that currencies are bought and sold in order to invest e.g. in short-term interest rates securities, in each country involved.

The main behavior of the currencies is generally manipulated by the corresponding government's economic policy expressed by the central bank, in the following two ways:

(i) Raising or lowering interest rates, thus making the country's securities more or less attractive.

(ii) Allowing the national currency to depreciate for some period of time (by supplying the foreign exchange market with massive quantities of its own currency), or causing it to

\footnotetext{
* Corresponding author.
} 
appreciate (by buying and thus absorbing large quantities from the market).

In each case, the objective is to stimulate either its exports (depreciation) or its imports (appreciation). Depending on the economic conditions, both objectives could be desirable and mirror each country's economic expectations. They induce, however, a considerable amount of noise in the data series of foreign currencies against the local one, which makes their mathematical analysis a difficult task.

Because of the factors mentioned above, a currency market is quite different from other traded markets. It is quite possible, if not certain, that this difference holds between currency markets of different countries.

Understanding exchange rates dynamics has important economic implications since flows and prices of tradable goods and international asset portfolios are closely related to these dynamics. However, the relevant literature examining the nature of price dependence in the foreign exchange spot, forward or futures markets has been contradictory. Standard time series (ARIMA) models and tests for unit roots are employed in some of these works (e.g. $[16,17,38])$ to show that exchange rates follow martingale processes. In a similar study Hsieh [24] finds no significant serial dependence in 4 different spot exchange rates series.

On the other hand, in another set of papers (e.g. $[25,26])$ the statistical properties of exchange rate changes are examined, the iid null hypothesis is rejected, thus putting in question a random walk explanation.

Finally, some studies look for long-range dependence in the data, in the form of irregular or nonperiodic cycles that cannot be captured by techniques assuming linear dependence (e.g. autocorrelation analysis). Long-term dependence of this form has been found in many time series in the fields of hydrology, meteorology and geophysics $[13,37]$. Similar dependence in financial series is incompatible with martingale models like random walks and according to Lo [30] can have important implications for many of the prevailing paradigms in financial economics.

A suitable framework for detecting long-range dynamics in time series is provided by rescale range analysis $(R / S)$, originated by Hurst [27,28] and further developed by Mandelbrot [33]. One of its valuable features is robustness to noise that renders it a very useful tool in analyzing noisy time series like exchange rates.

The empirical literature of $R / S$ analysis includes different studies with contradictory results. Classic $R / S$ analysis has been applied to employment series [4], gold market [6], stock market indices [43-47], common stock returns [19] and agricultural futures [21]. In all the above cases long-term memory has been reported.

On the other hand, a modified version of the $R / S$ statistic proposed by Phillips [48] and Lo [30] has been applied to macroeconomic data $[20,39]$, gold market prices [10], stock indices $[11,12,30,40]$ and common stock returns [22]. Weak (or absence) of long-term dependence has been reported by all of the above studies.

In the same context, studies using exchange rate data have also been contradictory. Booth et al. [5,7] and Peters [44] use classic $R / S$ analysis and find long memory or persistence in spot exchange rate data. On the contrary, Kao and $\mathrm{Ma}$ [29] find no long-term dependence in currency futures markets.

What is not clear is whether this controversy is due to the nature of the different data sets employed in terms of currencies, markets, sample size, noise level, etc., or to the testing framework that has been adopted. With respect to the latter it is interesting to notice that studies using classical $R / S$ analysis seem to support a long-term dependence hypothesis while the opposite seems to be the case for the modified $R / S$ statistic. Both these tests have certain advantages and disadvantages and suffer from problems that can seriously affect their results, in specific applications.

The purpose of this research is twofold. First, we shall try to provide a more complete framework for $R / S$ testing, enhanced by bootstrapping techniques 
that increase significantly the reliability of the empirical results.

Second, we shall use this framework to analyze four foreign exchange rate currencies against the Greek Drachma (GRD), namely the US Dollar (USD), the Deutsche Mark (DM), the French Franc (FF) and the British Pound (BP). Our goal is to detect long-term dynamics, i.e. to characterize each currency series in terms of its persistence, to reveal any existing cycles and estimate their average duration. Moreover, we shall exploit the ability of the $R / S$ method to distinguish between fractal noise processes and noisy chaos. Taking into account that, so far, the results of similar research have not yet established a general character of currency markets, this analysis is expected to be useful for comparison purposes as well.

\subsection{Methodology and Data}

Our time series data consist of the first differences of the natural logarithms of daily exchange rate prices of the four currencies mentioned in the Introduction, against Greek Drachma, which give returns in continuous time. The rates are those determined during the daily "fixing" sessions in the Bank of Greece, central bank of the country.

The data cover an 11-year period, from the 1st of January 1985 to the 31st of December 1995, consisting of 2660 daily observations. This amount of data is relatively small when compared to the time series used in the Natural Sciences, but large enough compared to other studies in Economics and Finance, for most of which the data used are much smaller.

Methodologically, a statistical description of the properties of the data is performed. Next, partial autocorrelograms and Schwartz's [51] information criterion are employed to test for the need of prefiltering.

Each currency is then tested for the existence of periodic or non-periodic cycles, through the application of the Rescaled Range and $V$-statistics analysis. Once an indication of a cycle is revealed, it is further tested to investigate whether its presence is a statistical artifact or it corresponds to a true cycle, using bootstrapping techniques against the Gaussian and the iid null hypotheses. In a last step, the modified $R / S$ test is applied to each currency and the results of this test are used to verify or put into question our findings from the classic $R / S$ analysis.

The rest of the paper is organized as follows: Section 2 includes a brief discussion of the classic $R / S$ analysis. Section 3 refers to some application problems that had to be dealt in order to have reliable results. A description of the modified $R / S$ analysis follows in Section 4 and its improved robustness over the classical test. Section 5 focuses on the empirical evidence of each currency separately. The results of the tests mentioned above are reported and their significance is evaluated. Finally, Section 6 presents a discussion on conclusions and remarks derived.

\section{THE $R / S$ ANALYSIS}

Technically, the origins of $R / S$ analysis are related to the " $T$ to the one-half rule", that is, to the formula describing the Brownian motion (BM):

$$
R=T^{0.5},
$$

where $R$ is the distance covered by a random particle suspended in a fluid and $T$ a time index. Equation (1) is commonly used in Finance to annualize volatility of e.g. monthly returns by multiplying monthly standard deviation by $12^{0.5}$. It is also obvious that (1) shows how $R$ is scaling with time $T$ in the case of a random system, and this scaling is given by the slope of the $\log (R)$ vs. $\log (T)$ plot, which is equal to 0.5 . Yet, when a system or a time series is not independent (i.e. not a random BM), (1) cannot be applied, so, Hurst gave the following generalization of (1) which can be used in this case:

$$
(R / S)_{n}=c n^{H},
$$

where $(R / S)_{n}$ is the Rescaled Range statistic measured over a time index $n, c$ is a constant and $H$ the Hurst exponent, which shows how the $R / S$ statistic is scaling with time. 
The objective of the $R / S$ method is to estimate the Hurst exponent, which, as we shall see, can characterize a time series. This can be done by transforming (2) to:

$$
\log (R / S)_{n}=\log (c)+H \log (n)
$$

and $H$ can be estimated as the slope of the $\log / \log$ plot of $(R / S)_{n}$ vs. $n$.

Given a time series $\left\{X_{t}: t=1, \ldots, N\right\}$, the $R / S$ statistic can be defined as the range of cumulative deviations from the mean of the series, rescaled by the standard deviation. The analytical procedure to estimate the $(R / S)_{n}$ values, as well as, the Hurst exponent by applying (3), is described in the following steps:

Step 1 The time period spanned by the time series of length $N$ is divided into $m$ contiguous subperiods of length $n$ such that $m * n=N$. The elements in each subperiod $X_{i, j}$ have two subscripts, the first $(i=1, \ldots, n)$ to denote the number of elements in each subperiod and the second $(j=1, \ldots, m)$ to denote the subperiod index. For each subperiod $j$ the $R / S$ statistic is calculated as:

$$
\begin{aligned}
\left(\frac{R}{S}\right)_{j}= & s_{j}^{-1}\left[\max _{1 \leq k \leq n} \sum_{i=1}^{k}\left(X_{i j}-\bar{X}_{j}\right)\right. \\
& \left.-\min _{1 \leq k \leq n} \sum_{i=1}^{k}\left(X_{i j}-\bar{X}_{j}\right)\right],
\end{aligned}
$$

where $s_{j}$ is the standard deviation for each subperiod.

In (4), the $k$ deviations from the subperiod mean have zero mean, hence the last value of the cumulative deviations for each subperiod will always be zero. Due to this, the maximum value of the cumulative deviations will always be greater or equal to zero, while the minimum value will always be less or equal to zero. Hence the range value (the bracketed term in (4)) will always be nonnegative.

Normalizing (rescaling) the range is important since it permits diverse phenomena and time periods to be compared, which means that $R / S$ analysis can describe time series with no characteristic scale.

Step 2 The $(R / S)_{n}$, i.e. the $R / S$ statistic for time length $n$, is given by the average of the $(R / S)_{j}$ values for all the $m$ contiguous subperiods with length $n$ as:

$$
\left(\frac{R}{S}\right)_{n}=\frac{1}{m} \sum_{j=1}^{m}\left(\frac{R}{S}\right)_{j}
$$

Step 3 Equation (5) gives the $R / S$ value which corresponds to a certain time interval of length $n$. In order to apply Eq. (3), steps 1 and 2 are repeated by increasing $n$ to the next integer value, until $n=$ $N / 2$, since, at least two subperiods are needed, to avoid bias.

From the above procedure, it becomes obvious that the time dimension is included in the $R / S$ analysis by examining whether the range of the cumulative deviations depends on the length of time used for the measurement. Once (5) is evaluated for different $n$ periods, the Hurst exponent can be estimated through an ordinary least square regression from (3).

The Hurst exponent takes values from 0 to 1 $(0 \leq H \leq 1)$. Gaussian random walks, or, more generally, independent processes, give $H=0.5$.

If $0.5 \leq H \leq 1$, positive dependence is indicated, and the series is called persistent or trend reinforcing and in terms of Eq. (1), the system covers more distance than a random one. In this case the series is characterized by a long memory process with no characteristic time scale. The lack of characteristic time scale (scale invariance) and the existence of a power law (the $\log / \log$ plot) are the key characteristics of a fractal series.

If $0 \leq H \leq 0.5$, negative dependence is indicated, yielding anti-persistent or mean-reverting behavior. ${ }^{\dagger}$ In terms of Eq. (1), the system covers less distance than a random series, which means that it reverses itself more frequently than a random process.

\footnotetext{
${ }^{\dagger}$ Only if the system under study is assumed to have stable mean.
} 
A Hurst exponent different from 0.5 may characterize a series as fractal. However, a fractal series might be the output of different kinds of systems. A "pure" Hurst process is a fractional Brownian motion [34-36], also known as biased random walk or fractal noise or colored noise, that is, a random series the bias of which can change abruptly but randomly in direction or magnitude.

Chaotic systems have also Hurst exponents $H>0.5$, and in chaotic terms long memory effects correspond to sensitive dependence on initial conditions. Actually, the latter property combined with fractality characterizes chaotic systems. Pure chaotic processes have Hurst exponents close to 1 .

When dealing, however, with real data the problem of distinguishing between the above alternatives becomes more difficult due to the existence of noise. Most series are contaminated by either additive or dynamical (system) noise. Hence, in most cases, including financial data, the problem is to distinguish between fractal noise and noisy chaos.

$R / S$ analysis provides a very useful tool to solve the above problem since it is extremely robust to both additive and system noise [47]. Noise lowers the $H$ value of a series and obscures the difference, e.g. between a fractional Brownian motion with $H=0.70$ and a chaotic process which originally has $H=0.92$ but noise contamination reduces it to 0.70 , as well. However, $R / S$ analysis is able to detect, even when noise is present, the existence of cycles in the series and thus to characterize it.

Cycles can either be periodic or non-periodic in the sense that the system has no absolute frequency. Non-periodic cycles can be further divided to statistical cycles and chaotic cycles. Fractal noises exhibit statistical cycles, i.e. cycles with no average cycle length. Actually, they are random cycles of different length due to long-run correlations and randomly changing bias ${ }^{\ddagger}$ of the system. On the contrary, deterministic systems like chaotic flows or noisy chaotic processes have chaotic cycles which have an average frequency.
In general, fractal noises will have no discernible cycles, but in practice and in a certain time scale, fractional Brownian motion might exhibit a finite memory effect, which is usually a statistical artifact due to the limited length of the series examined. In this case, fractal noise can be distinguished from a chaotic alternative by examining whether the cycle is independent of the time scale used. Cycles independent of the time scale indicate the (noisy) chaos alternative. Detection of cycles and estimation of their length can be accomplished by the use of the $V$-statistic $[28,47]$ defined as:

$$
V_{n}=(R / S)_{n} / n^{0.5}
$$

The $V_{n}$ vs. $\log (n)$ plot gives a flat line for an independent random process and an upwardly sloping curve in the case of persistent series. The existence of a cycle and its length can be discerned (even when noise is present) from the "break-point" in this plot occurring when $V_{n}$ reaches a peak and then flattens out, an indication that the longmemory process has dissipated.

\section{APPLICATION PROBLEMS}

Some problems should be discussed with respect to the application of classic $R / S$ analysis. A first problem is related to the procedure of step 1 described above. In order to create the $m$ subperiods, the series of $N$-length, should be divided by $n$ (the length of each subperiod) as:

$$
m=N / n \text {. }
$$

In (7), $m$ should always be an integer, since all the subperiods must have the same length $n$. Since $N$ is fixed and $n$ is changing, some points should be left out when (7) gives no integer $m$. Obviously, the number of these "left-out" points depends on the length of the data $N$, and the divisor $n$, and in some cases exceeds $30 \%$ or more of the total points in the

\footnotetext{
${ }^{\ddagger}$ Abrupt changes in direction due to exogenous events, predictable or not.
} 
data resulting to significant bias and unreliable $H$ estimates. To account for this, Peters [47] suggests that only the time increments that include both the beginning and the ending points of the data should be used i.e. only the $n$ values that produce integer $m$ values. However, these $n$ values might be very few, especially when the $N$-length is small which means that there are not enough regressors to estimate $H$ in (3). Hence, for small data sets there is a "tradeoff" between adequate number of regressors and bias induced by "left-out" points. To account for this, the $N$-length is adjusted in each case, to give the maximum number of perfect divisors and a very small number of "left-out" points (less than $2 \%$ ), in order to minimize bias.

A second problem is the evaluation of the $H$ exponent from a statistical point of view. Specifically, we should be able to assess whether an $H$ value is statistically significant in comparison to a random null, i.e. to the $H$ exponent exhibited by an independent random system. Peters [47] shows that under the Gaussian null, a modification of a formula developed by Anis and Lloyd [2] allows for hypothesis testing by computing $E(R / S)_{n}$ and $E(H)$, the expected variance of which will depend only on the total sample size $N$, as $\operatorname{Var}(H)=1 / N$.

However, if the null is still iid randomness but not Gaussianity, the formal hypothesis testing is not possible. To overcome this problem we used bootstrapping [14] to assess the statistical significance of the $H$ exponents of our series, against both the Gaussian and the iid random null hypotheses.

To test against the Gaussian random null, the $H$ exponent from 5000 random shuffles of a Gaussian random surrogate, having the same length, mean and variance with our return series is calculated and compared to the test statistic i.e. the actual $H$ exponent of our series. If the latter is found to be greater than 0.5 , and persistence of the series is possible, then the null hypothesis tested is formed as: $H_{0}: H=H_{\mathrm{G}}$ and the alternative $H_{1}: H>H_{\mathrm{G}}$ or in words: "The actual $H$ estimate from the series tested is significantly different (greater) than the $H_{\mathrm{G}}$ estimate from a Gaussian random data with the same length, mean and std as the series tested".

The significance level of the test is constructed as the frequency with which the pseudostatistic (the $H_{\mathrm{G}}$ estimate) from the Gaussian shuffles is greater than or equal to the actual statistic for the tested (unshuffled) data [42]. The null hypothesis is rejected if the significance level is smaller than the conventional rejection levels of $1 \%, 2.5 \%$ or $5 \%$.

When the actual $H$ statistic is found to be lower than 0.5 and anti-persistence is possible, the null can be formed again as: $H_{0}: H=H_{\mathrm{G}}$ but the alternative this time is $H_{1}: H<H_{\mathrm{G}}$. In this case, the significance level of the test is constructed as the frequency with which the pseudostatistic $H_{\mathrm{G}}$ is smaller than or equal to the actual statistic and the null is rejected if the significance level is smaller than the conventional rejection levels of $1 \%, 2.5 \%$ or $5 \%$.

To test against the iid null, the same procedure is followed but this time we randomize the series tested to produce 5000 iid random samples having the same length and distributional characteristics as the original series. In this case, rejection of the null means that the actual $H$ exponent calculated from the original series is significantly different (greater or smaller depending on the hypothesis tested) from the one calculated from an iid random series. Hence, this is also a test for non-iid-ness.

Finally, a third problem is related to the sensitivity of $R / S$ analysis to short-term dependence which can lead to unreliable results $[3,4,20$, 30,31,41]. Peters [47] shows that Autoregressive (AR), Moving Average (MA) and mixed ARMA processes exhibit Hurst effects, but once short-term memory is filtered out by an $\operatorname{AR}(1)$ specification, these effects cease to exist. On the contrary, ARCH and GARCH models do not exhibit long-term memory and persistence effects at all. Hence, a series should be pre-filtered for short-term linear dependence before to apply the $R /$ $S$ analysis. In our analysis, we use partial autocorrelograms and Schwartz's information criterion to indicate the best-fit time series linear model to our data. 


\section{THE MODIFIED $R / S$ STATISTIC}

An alternative way to account for short-term dependence is to use the $R / S$ test statistic modified by Lo [30]. In Lo's modification, short-range dependence is incorporated into the partial sum variance estimator to the denominator of the classical Mandelbrot's [33] $R / S$ statistic as:

$$
\begin{aligned}
& Q_{q}=\hat{s}_{N}^{-1}(q)\left[\max _{1 \leq k \leq N} \sum_{t=1}^{k}\left(X_{t}-\bar{X}_{N}\right)\right. \\
&\left.-\min _{1 \leq k \leq N} \sum_{t=1}^{k}\left(X_{t}-\bar{X}_{N}\right)\right],
\end{aligned}
$$

where

$$
\begin{gathered}
\hat{s}_{N}^{2}(q)=\hat{s}_{x}^{2}+2 \sum_{t=1}^{q} w_{t}(q) \hat{g}_{t}, \\
w_{t}(q)=1-\frac{t}{q+1}, \quad q<N, \\
\hat{s}_{N}^{2}(q)=\frac{1}{N} \sum_{t=1}^{N}\left(X_{t}-\bar{X}_{N}\right)^{2} \\
+\frac{2}{N} \sum_{t=1}^{q} w_{t}(q)\left\{\sum_{l=k+1}^{N}\left(X_{l}-\bar{X}_{N}\right)\left(X_{l-k}-\bar{X}_{N}\right)\right\}
\end{gathered}
$$

and $\hat{s}_{x}^{2}$ and $\hat{g}_{t}$ being the variance and autocovariance estimators of $X$.

According to (9) and (10), if $\left\{X_{t}\right\}$ is subject to short-range dependence, the estimator $\hat{s}_{N}(q)$ involves the sums of squared deviations of $X$ and its weighted autocovariances up to lag $q$. This latter term consists the modification of the original $(R / S)_{N}$ statistic.

This test, unlike classic $R / S$ analysis described above, does not have to rely on subsample analysis.
The test's null is short-term dependence which operationally is defined by Lo as a "strong-mixing" process, a notion due to Rosenblatt [50]" in order to derive the asymptotic distribution of $Q_{q}$.

Lo shows that under certain conditions which place restrictions on the maximal moments, the degree of distributional heterogeneity and the maximal degree of dependence in $\left\{X_{t}\right\}$, the statistic $V_{q}=N^{-(1 / 2)} Q_{q}$ converges to the range of a "Brownian bridge" on the unit interval, a well defined random variable with mean $(\pi / 2)^{(1 / 2)}$, variance $\pi^{2} /$ $6-\pi / 2$ and a positively skewed distribution function.

The critical values of the test derived by the asymptotic cumulative distribution function are given in Table I.

The main advantage of the test is that it allows for formal statistical testing and is robust against serial correlation and some forms of non-stationarity. It is specifically designed to distinguish between weakly dependent processes (e.g. ARMA) and strongly dependent processes (e.g. fractionally integrated (ARFIMA) models [18,23]). Notice that the main characteristic of these strongly dependent processes is the slowly decaying autocorrelation functions and non-periodic cyclical patterns. Moreover, the test's null is wide enough to include models with longer-term correlations like the stochastic models of persistence proposed by Campbell and Mankiw [9], Fama and French [15] and Poterba and Summers [49].

The main disadvantage of the test is that unlike the classic $R / S$ analysis is not able to specify the cycle length of the series tested. There are, in addition, certain shortcomings related to the test "per se". Lo [30] shows that there are forms of short-term dependence violating the assumptions of the test's null. ${ }^{\S} \mathrm{He}$ also reports low power of

TABLE I Asymptotic critical values of the modified $R / S$ statistic

\begin{tabular}{lcccccccc}
\hline Probability level & $0.5 \%$ & $2.5 \%$ & $5 \%$ & $10 \%$ & $90 \%$ & $95 \%$ & $97.5 \%$ & $99.5 \%$ \\
Critical value & 0.721 & 0.809 & 0.861 & 0.927 & 1.620 & 1.747 & 1.862 & 2.098 \\
\hline
\end{tabular}

\footnotetext{
IStrong mixing requires that the maximal dependence between two events becomes trivially small as their separation time increases without bound.

${ }^{\S}$ For example, the test has no power against processes with maximal moments less than 4 violating the moment condition of the test, or the first difference of a stationary process violating the heterogeneity condition.
} 
the test against chaotic processes like the "tentmap", a long-range dependent process with very low autocorrelation.

Hiemstra and Jones [22] find that right- and left-tailed bootstrapped critical values of the modified $R / S$ statistic, fall below their asymptotic counterparts, resulting to higher right-tailed and lower left-tailed rejection rates. According to their analysis, this is due to the test sensitivity to moment condition failure, i.e. to the magnitude of the maximal moment of their series which is less than 4 . Brock and de Lima [8] use Monte Carlo simulation to find also that the sampling distribution of the test is shifted to the left, relatively to the asymptotic distribution.

Another problem is related to the sensitivity of the test to the truncation lag-parameter $q$ in Eq. (8). Lo [30] employs Monte Carlo simulations to assess the power of the test which declines with increasing $q$ and decreasing sample size. In fact, even for sample sizes of $N=1000$, the empirical rejection rates were much lower than nominal sizes for $q$ values exceeding $N^{1 / 3}$.

Little is known about the optimal choice of $q$, although Andrews [1] suggests a data-dependent formula given by:

$$
q_{N}=1+\operatorname{INT}\left[\left(\frac{3 N}{2}\right)^{1 / 3} \cdot\left(\frac{2 \hat{\boldsymbol{r}}}{1-\hat{\boldsymbol{r}}}\right)^{2 / 3}\right]
$$

where, $N$ is the data length and $\hat{r}$ the estimated first order autocorrelation of the data. However, the truncation lag given by this formula is optimal only for an AR(1) data generating process.
Although $R / S$ analysis combined with bootstrapping for assessing the statistical significance of the $H$ exponent provides a powerful tool for detecting persistent behavior and long-term cycles, we employed also the modified $R / S$ statistic to cross-check our findings. In our application of the modified $R / S$ statistic, we used different $q$-lengths equal to $q_{n}=\operatorname{INT}\left[N^{1 / 4}\right], \operatorname{INT}\left[N^{1 / 3}\right]$, INT $\left[N^{1 / 2}\right]$, as well as, the $q$ values derived from the datadependent formula in (11). In addition, to assess the test's results, we have used the asymptotic and bootstrapped critical values of our series. The latter are based on the test statistics derived from 5000 time-scrambled shuffling of our data, producing iid series consistent with the test's null and robust to violations of the moment condition of the test.

\section{EMPIRICAL EVIDENCE}

\section{The USD/GRD Exchange Rate}

The basic statistical properties and the time series plot $^{\#}$ of the Dollar/Drachma exchange rate return series are listed in Table II and Fig. 1 respectively.

This statistical profile shows a non-normal skewed and leptokurtic series. Partial autocorrelogram and Schwartz's information criterion indicate no significant autocorrelation, hence no pre-filtering is necessary and $R / S$ analysis can be applied to the original return series.

In Fig. 2(a) and (b), the $\log -\log$ plot of the $R / S$ statistic versus time ( $N$-days) and the semi-log plot of the $V$-statistic respectively show a possible cycle with a length of 870 trading days. The Hurst

TABLE II Statistical description of the USD/GRD daily return series

\begin{tabular}{lclr}
\hline Sample size & 2659 & Range & 0.085509 \\
Average & 0.000101 & Lower quartile & -0.00159 \\
Median & 0.000026 & Upper quartile & 0.001715 \\
Variance & 0.00001 & Skewness & 3.85 \\
Standard deviation & 0.003223 & Standardized skewness & 81.19 \\
Minimum & -0.01735 & Kurtosis & 76.81 \\
Maximum & 0.068159 & Standardized kurtosis & 3186.65 \\
\hline
\end{tabular}

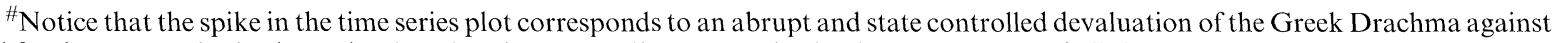
all foreign currencies in the early 1985 . So, the same spike appears in the time series plots of all the currencies analyzed in this paper. Notice that this outlayer does not affect our results which hold even if we use for our analysis the time period after the devaluation date.
} 


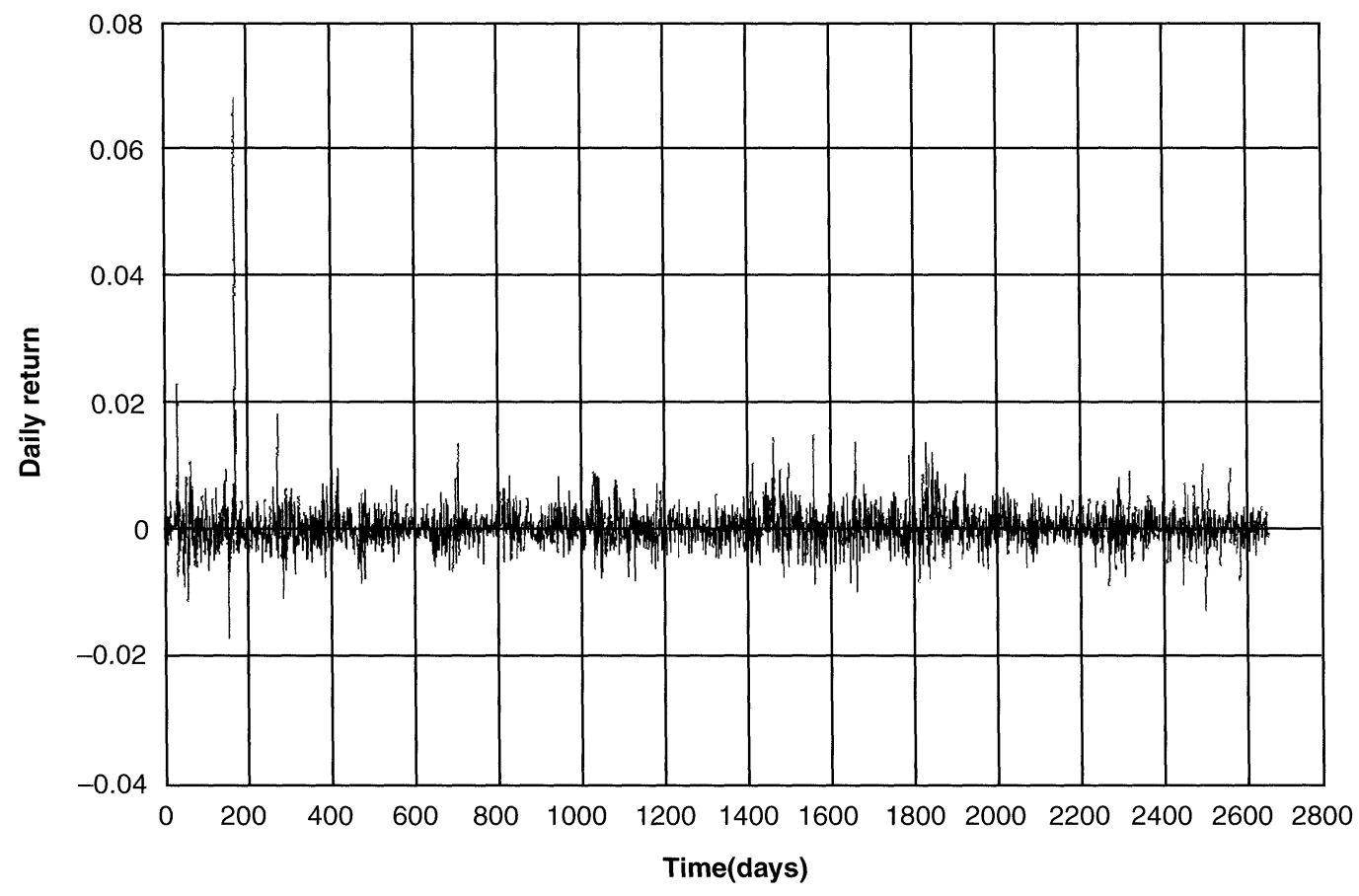

FIGURE 1 Time series plot of the daily returns for the USD/GRD exchange-rate.

USD/GRD - One day returns
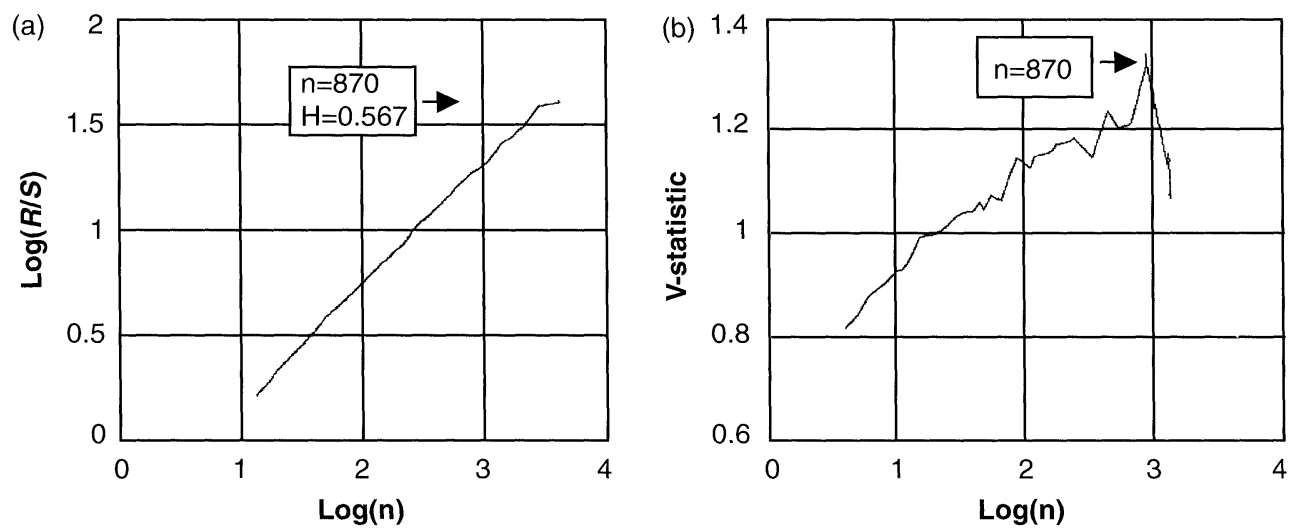

FIGURE $2 R / S$ analysis of the USD/GRD daily returns (a) Hurst exponent estimation through the $R / S$ statistic plot (b) Cyclelength estimation through the V-statistic plot.

exponent corresponding to this cycle length is 0.57 , a low value indicating noisy series. Indeed, by using bootstrapping to assess the statistical significance of this Hurst estimate, we found that the null hypotheses including the Gaussian random and the random iid alternatives are not rejected since the significance level of the test in both cases was greater than $5 \%$, as Table III shows. This means that the alleged cycle was just a statistical artifact and not a true cycle. 
TABLE III Hurst estimates and test of significance against two random alternatives of the daily USD/ GRD returns

\begin{tabular}{lcc}
\hline A. $R / S$ analysis results & $4<\mathrm{n}<870$ & $4<\mathrm{n}<1320$ \\
Hurst $(\mathrm{H})$ exponent & $\begin{array}{c}\text { Cycle length) } \\
0.567\end{array}$ & $\begin{array}{c}\text { (Total data length) } \\
0.549\end{array}$ \\
B. Bootstrapping results & $4<\mathrm{n}<870$ & $4<\mathrm{n}<1320$ \\
& $($ Cycle length) & \\
B1. $H_{0}: H>H_{\mathrm{G}}($ Gaussian random alternative) & & 0.541 \\
Mean $H_{\mathrm{G}}$ value & 0.548 & $40.1 \%$ \\
Significance level & $24.4 \%$ & \\
B2. $H_{0}: H>H_{\mathrm{R}}$ (iid random alternative) & & 0.540 \\
Mean $H_{\mathrm{G}}$ value & 0.546 & $38.1 \%$ \\
Significance level & $22.3 \%$ & \\
\hline
\end{tabular}

TABLE IV The modified $R / S$ statistic of the daily USD/GRD returns and bootstrapped critical values of the test statistic

\begin{tabular}{lcccr}
\hline$q$ & $\begin{array}{c}\text { Andrew's } \\
1\end{array}$ & $\mathrm{~N}^{1 / 4}$ & $\mathrm{~N}^{1 / 3}$ & $\begin{array}{c}\mathrm{N}^{1 / 2} \\
51\end{array}$ \\
\hline$V_{q^{-} \text {-statistic }}$ & 0.940 & 0.924 & 0.934 & 0.958 \\
Bootstrapped critical values & & & & \\
Significance level & & & & \\
$1.0 \%$ & 1.949 & 1.939 & 1.888 & 1.875 \\
$2.5 \%$ & 1.817 & 1.790 & 1.780 & 1.765 \\
$5.0 \%$ & 1.720 & 1.708 & 1.710 & 1.682 \\
$10.0 \%$ & 1.572 & 1.565 & 1.571 & 1.556 \\
\hline
\end{tabular}

However, fractality, long-term memory (with no average cycle) and persistence of the series cannot be ruled out, unless the Hurst estimate of the total series is tested too against the random iid null. As Table III shows the Hurst exponent corresponding to the total length of the series is 0.55 , a value which is not significantly different (at a significant level less than 1\%) from the mean bootstrapped value of the Hurst exponent derived from 5000 random shuffles of the original series. Hence, the null of a random series with no longterm memory effects cannot be rejected and the alternative of a fractional Brownian motion (black noise) with an infinite memory seems implausible.

The results from the modified $R / S$ statistic seem to confirm the above findings. Table IV, presents the estimates of the $V_{q}$ statistic for different $q$ values. We used both the asymptotic values, as well as, the bootstrapped critical values to assess the statistical significance of the test statistic. The righttail bootstrapped critical values (at the $1 \%, 2.5 \%$, $5 \%$ and $10 \%$ significance levels) corresponding to each one of the $q$ values are also presented in the lower part of Table IV. These values are lower than the asymptotic ones, confirming the findings of Hiemstra and Jones [22]. It is obvious that for all the $q$ values the $V_{q}$ estimates do not reject the test's short-range dependence null when compared to either the asymptotic or the bootstrapped critical values of the test.

\section{The BP/GRD Exchange Rate}

The statistical properties of the British Pound/ Drachma exchange rate return series presented in Table V, exhibit similar distributional characteristics to the USD/GRD returns, with even larger values for skewness and kurtosis. The time series plot of the series in Fig. 3 also shows no apparent structure.

In this case too, partial autocorrelogram and Schwartz's information criterion indicate no significant autocorrelation and the data is not pre-filtered. 
TABLE V Statistical description of the BP/GRD daily return series

\begin{tabular}{lclr}
\hline Sample size & 2659 & Range & 0.082554 \\
Average & 0.00015 & Lower quartile & -0.00085 \\
Median & 0.000139 & Upper quartile & 0.001084 \\
Variance & 0.000006 & Skewness & 7.97 \\
Standard deviation & 0.002455 & Standardized skewness & 167.88 \\
Minimum & -0.01434 & Kurtosis & 224.29 \\
Maximum & 0.068214 & Standardized kurtosis & 2360.83 \\
\hline
\end{tabular}

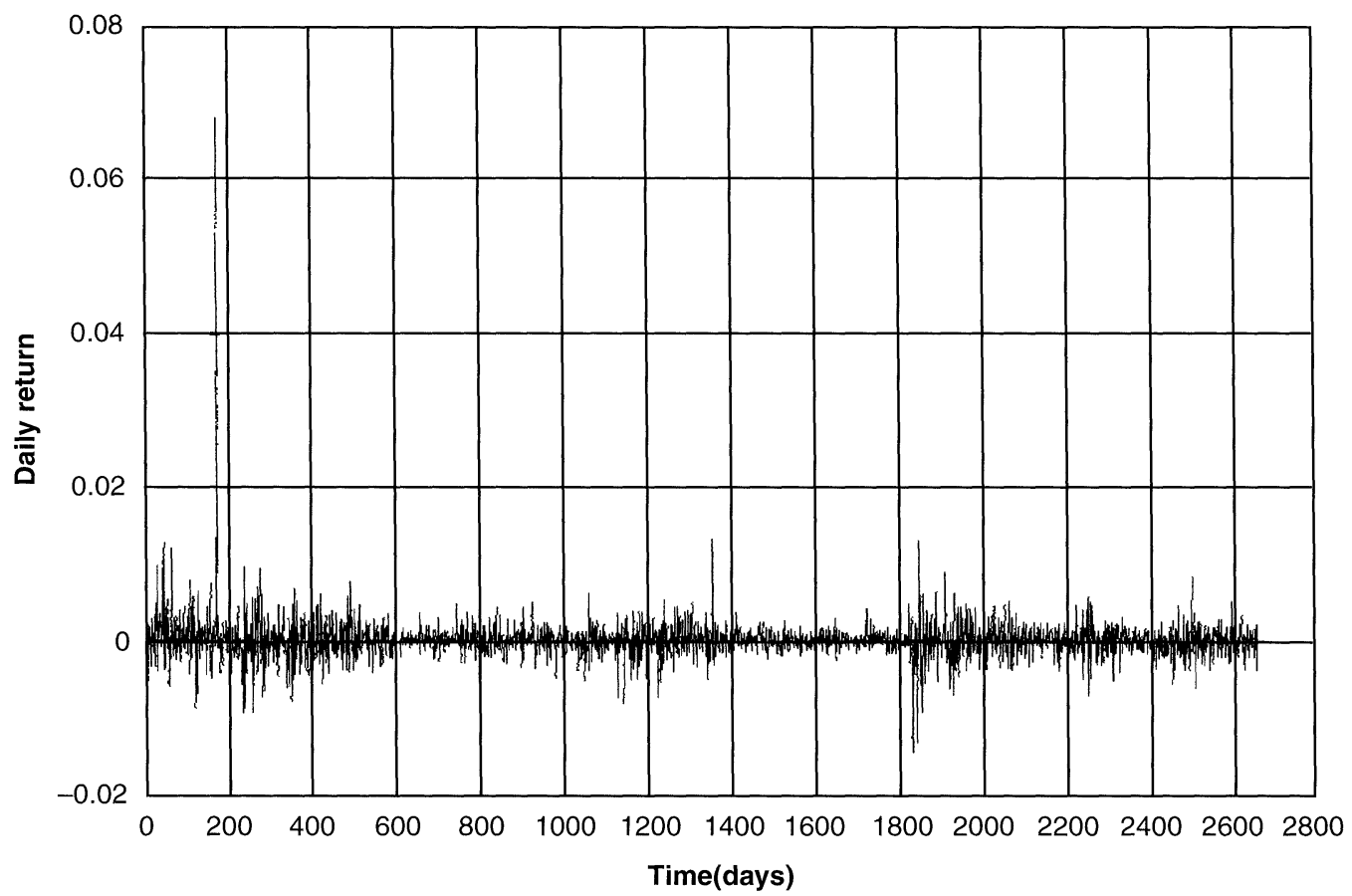

FIGURE 3 Time series plot of the daily returns for the BP/GRD exchange-rate.

The structure of the two series looks the same in terms of $R / S$ analysis as well. The $\log -\log$ plot of the $R / S$ statistic versus time ( $N$-days) and the semi$\log$ plot of the $V$-statistic in Fig. 4(a) and (b) respectively show again a possible cycle of approximately 870 trading days with a slightly larger Hurst estimate of 0.573 . However, bootstrapping results in Table VI, show that this estimate is not a significant one, compared to both the Gaussian and iid random nulls although the significance levels are lower than the previous case. The fractal Brownian motion alternative is also not accepted since the Hurst estimate of the total series ( $H=$ 0.571) was not found to be significant as Table VI presents.
Results from the modified $R / S$ statistic are presented in Table VII. The short-term dependence null is not rejected even at a $10 \%$ significance level by the use of both the asymptotic and the bootstrapped critical values of the test statistic, for all the different $q$ values used.

\section{The DM/GRD Exchange Rate}

The statistical properties of the series are presented in Table VIII showing, as in the previous cases, deviation from the normal distribution. However, in this case standard deviation of the series is lower, while skewness and kurtosis are much more pronounced. These structural differences are 
BP/GRD - One day returns
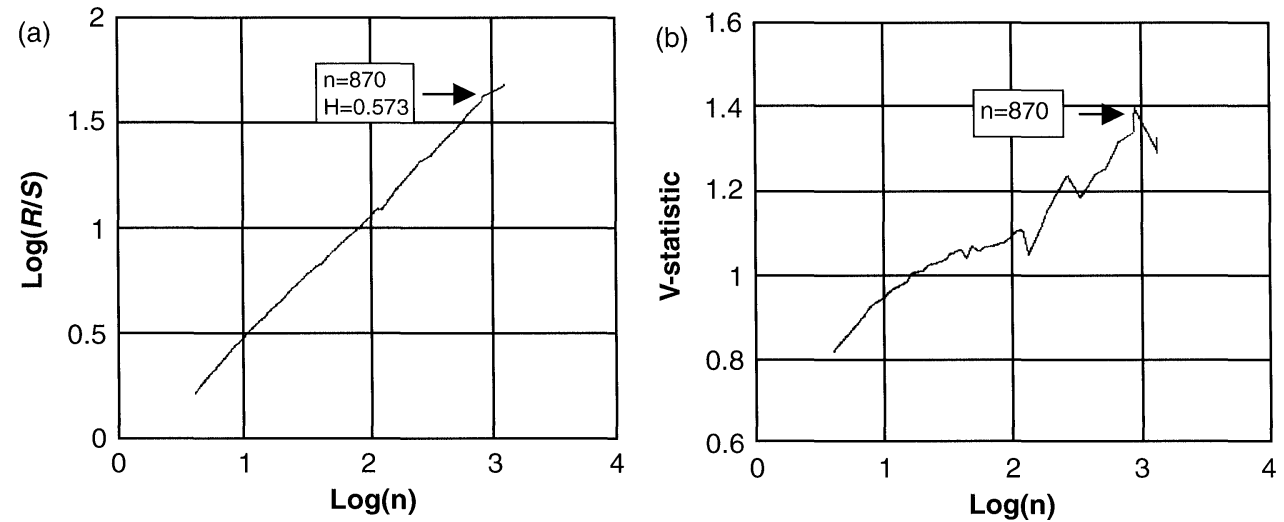

FIGURE $4 R / S$ analysis of the BP/GRD daily returns (a) Hurst exponent estimation through the $R / S$ statistic plot (b) Cyclelength estimation through the V-statistic plot.

TABLE VI Hurst estimates and test of significance against two random alternatives of the daily BP/GRD returns

\begin{tabular}{|c|c|c|}
\hline A. $R / S$ analysis results & $\begin{array}{c}4<\mathrm{n}<870 \\
\text { (Cycle length) }\end{array}$ & $\begin{array}{c}\quad 4<\mathrm{n}<1320 \\
\text { (Total data length) }\end{array}$ \\
\hline Hurst $(\mathrm{H})$ exponent & 0.573 & 0.571 \\
\hline B. Bootstrapping results & $\begin{array}{c}4<\mathrm{n}<870 \\
\text { (Cycle length) }\end{array}$ & $\begin{array}{c}\quad 4<\mathrm{n}<1320 \\
\text { (Total data length) }\end{array}$ \\
\hline $\begin{array}{l}\text { B1. } H_{0}: H>H_{\mathrm{G}}(\text { Gaussian random alternative }) \\
\text { Mean } H_{\mathrm{G}} \text { value } \\
\text { Significance level }\end{array}$ & $\begin{array}{c}0.549 \\
19.6 \%\end{array}$ & $\begin{array}{c}0.542 \\
18.2 \%\end{array}$ \\
\hline $\begin{array}{l}\text { B2. } H_{0}: H>H_{\mathrm{R}} \text { (iid random alternative) } \\
\text { Mean } H_{\mathrm{R}} \text { value } \\
\text { Significance level }\end{array}$ & $\begin{array}{c}0.545 \\
14.6 \%\end{array}$ & $\begin{array}{c}0.539 \\
13.1 \%\end{array}$ \\
\hline
\end{tabular}

TABLE VII The modified $R / S$ statistic of the daily BP/GRD returns and bootstrapped critical values of the test statistic

\begin{tabular}{lcccc}
\hline$q$ & $\begin{array}{c}\text { Andrew's } \\
1\end{array}$ & $\begin{array}{c}\mathrm{N}^{1 / 4} \\
7\end{array}$ & $\begin{array}{c}\mathrm{N}^{1 / 2} \\
14\end{array}$ & $\begin{array}{c}\mathrm{N}^{1 / 2} \\
51\end{array}$ \\
\hline$V_{q}$-statistic & 1.309 & 1.301 & 1.296 & 1.235 \\
Bootstrapped critical values & & & & \\
Significance level & & & & \\
$1.0 \%$ & 1.875 & 1.853 & 1.837 & 1.788 \\
$2.5 \%$ & 1.770 & 1.757 & 1.760 & 1.684 \\
$5.0 \%$ & 1.672 & 1.652 & 1.647 & 1.603 \\
$10.0 \%$ & 1.560 & 1.559 & 1.541 & 1.511 \\
\hline
\end{tabular}

discernible in the time series plot in Fig. 5, where the maximum value, as in the previous cases, corresponds to the abrupt devaluation of drachma mentioned before.

Both the partial autocorrelogram and Schwartz's information criterion indicate first order autocorrelation, so an $\mathrm{AR}(1)$ specification is employed to pre-filter the series.

$R / S$ analysis is performed to the filtered series and the $\log -\log$ plot of the $R / S$ statistic versus time ( $N$-days) and the semi-log plot of the $V$-statistic are shown in Fig. 6(a) and (b), respectively. This time a 
EXCHANGE RATES

TABLE VIII Statistical description of the DM/GRD daily return series

\begin{tabular}{lclr}
\hline Sample size & 2659 & Range & 0.088162 \\
Average & 0.00023 & Lower quartile & -0.00016 \\
Median & 0.000108 & Upper quartile & 0.00051 \\
Variance & 0.0000028 & Skewness & 24.53 \\
Standard deviation & 0.001682 & Standardized skewness & 516.38 \\
Minimum & -0.02059 & Kurtosis & 986.96 \\
Maximum & 0.067572 & Standardized kurtosis & 10388.60 \\
\hline
\end{tabular}

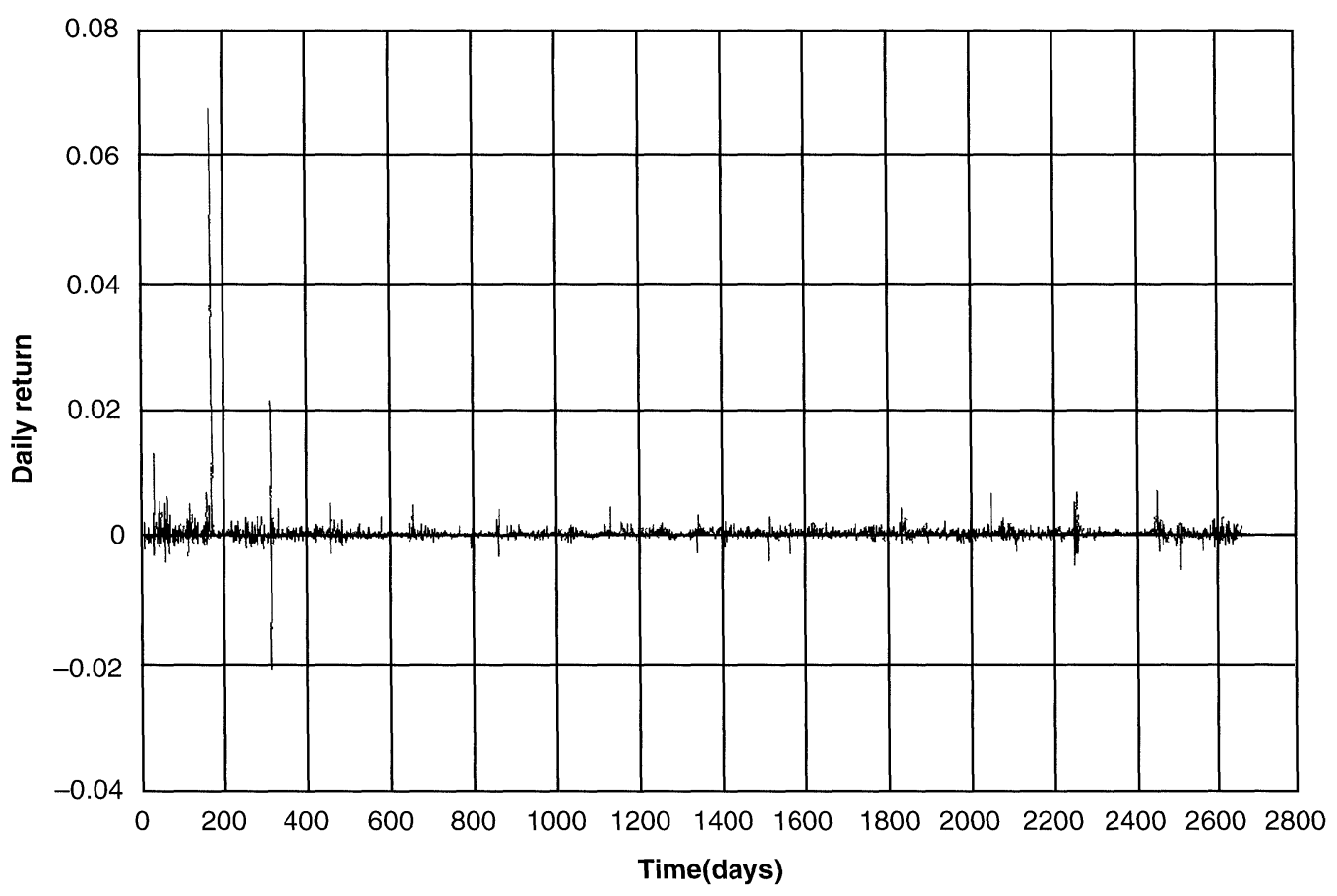

FIGURE 5 Time series plot of the daily returns for the DM/GRD exchange-rate.

DM/GRD - One day returns
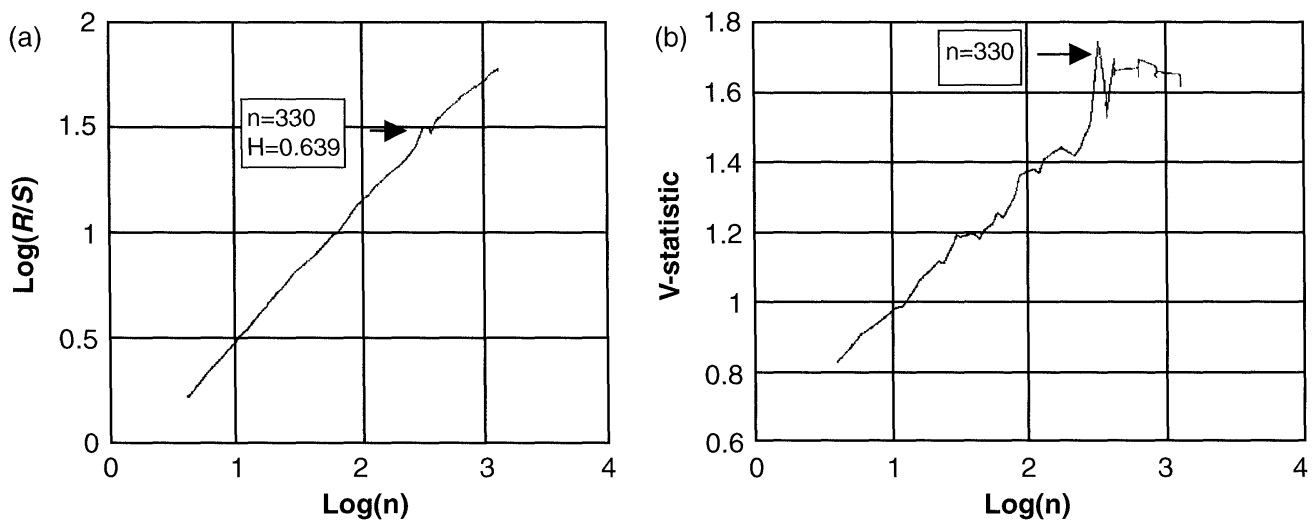

FIGURE $6 \quad R / S$ analysis of the DM/GRD AR(1)-filtered daily returns (a) Hurst exponent estimation through the $R / S$ statistic plot (b) Cycle-length estimation through the V-statistic plot. 
much shorter cycle of 330 trading days is clearly discernible with a Hurst estimate of $H=0.639$. Bootstrapping results in Table IX show that this estimate is highly significant (at $1 \%$ significance level), compared to both the Gaussian and iid random nulls. The third column in Table IX presents the Hurst estimate for the remaining data length i.e. the slope of the $R / S$ curve in Fig. 6(a) after the break point. The estimated Hurst exponent drops to $H=0.535$ and as bootstrapping results show, it cannot be distinguished from the Hurst estimate of a random process either Gaussian or iid. So, after the 330-day cycle the process becomes (the slope crosses over to) a random walk.

When the modified $R / S$ statistic is applied, the long-term dependence alternative is not ruled out especially when the bootstrapped critical values are used. As Table X shows, the null of short-term dependence is not rejected (at the conventional significance levels) only when $q=n^{1 / 2}$ and the asymptotic critical values are used to assess the significance of the $V_{q}$ statistic.

The remaining step is to investigate whether the cycle that has been found is a true non-periodic cycle compatible with a noisy chaos explanation or a stochastic boundary due to data size compatible with fractional Brownian motion with finite memory. A cycle independent of the sample size is a clear indication towards the first alternative [47]. So, $R / S$ analysis is performed to 5-day and 20-day returns produced from our daily data. Both series were not pre-filtered since no autocorrelation was found.

Figures 7(a), (b) and 8(a), (b) display that 5-day returns and 20-day returns exhibit clear cycles of approximately 65 and 15 observations respectively, both equivalent to the 330 one-day cycle.

As expected from non-white noise data, the $H$ exponents corresponding to the above cycles (see Table XI) are increasing with longer sampling intervals, due to the less noisy character of lower frequency data. These cycles are not artifacts since

TABLE IX Hurst estimates and test of significance against two random alternatives of the daily DM/GRD returns

\begin{tabular}{|c|c|c|}
\hline A. $R / S$ analysis results & $\begin{array}{l}4<\mathrm{n}<330 \\
\text { (Cycle length) } \\
\quad 0.639\end{array}$ & $\begin{array}{c}330<\mathrm{n}<1320 \\
\text { (Remaining data length) } \\
0.535\end{array}$ \\
\hline B. Bootstrapping results & $\begin{array}{c}4<\mathrm{n}<330 \\
\text { (Cycle length) }\end{array}$ & $\begin{array}{c}330<\mathrm{n}<1320 \\
\text { (Remaining data length) }\end{array}$ \\
\hline $\begin{array}{l}\text { B1. } H_{0}: H>H_{\mathrm{G}} \text { (Gaussian random alternative) } \\
\text { Mean } H_{\mathrm{G}} \text { value } \\
\text { Significance level }\end{array}$ & $\begin{array}{l}0.562 \\
0.1 \%\end{array}$ & $\begin{array}{c}0.510 \\
40.4 \%\end{array}$ \\
\hline $\begin{array}{l}\text { B2. } H_{0}: H>H_{\mathrm{R}}(\text { iid random alternative }) \\
\text { Mean } H_{\mathrm{R}} \text { value } \\
\text { Significance level }\end{array}$ & $\begin{array}{l}0.552 \\
0.1 \%\end{array}$ & $\begin{array}{c}0.500 \\
38.7 \%\end{array}$ \\
\hline
\end{tabular}

TABLE X The modified $R / S$ statistic of the daily DM/GRD returns and bootstrapped critical values of the test statistic

\begin{tabular}{lcccc}
\hline$q$ & Andrew's & $\mathrm{N}^{1 / 4}$ & $\mathrm{~N}^{1 / 3}$ & $\mathrm{~N}^{1 / 2}$ \\
& 5 & 7 & 14 & 51 \\
\hline$V_{q}$-statistic & 1.884 & 1.861 & 1.757 & 1.550 \\
Level of significance (asymptotic values) & $2.5 \%$ & $2.5 \%$ & $5 \%$ & $>10 \%$ \\
Level of significance (bootstrapped values) & $1 \%$ & $1 \%$ & $2.5 \%$ & $5 \%$ \\
Bootstrapped critical values & & & & \\
Significance level & & & & \\
$1.0 \%$ & 1.755 & 1.730 & 1.743 & 1.680 \\
$2.5 \%$ & 1.672 & 1.648 & 1.625 & 1.602 \\
$5.0 \%$ & 1.541 & 1.550 & 1.528 & 1.505 \\
$10.0 \%$ & 1.443 & 1.447 & 1.445 & 1.411 \\
\hline
\end{tabular}


DM/GRD - Five day returns
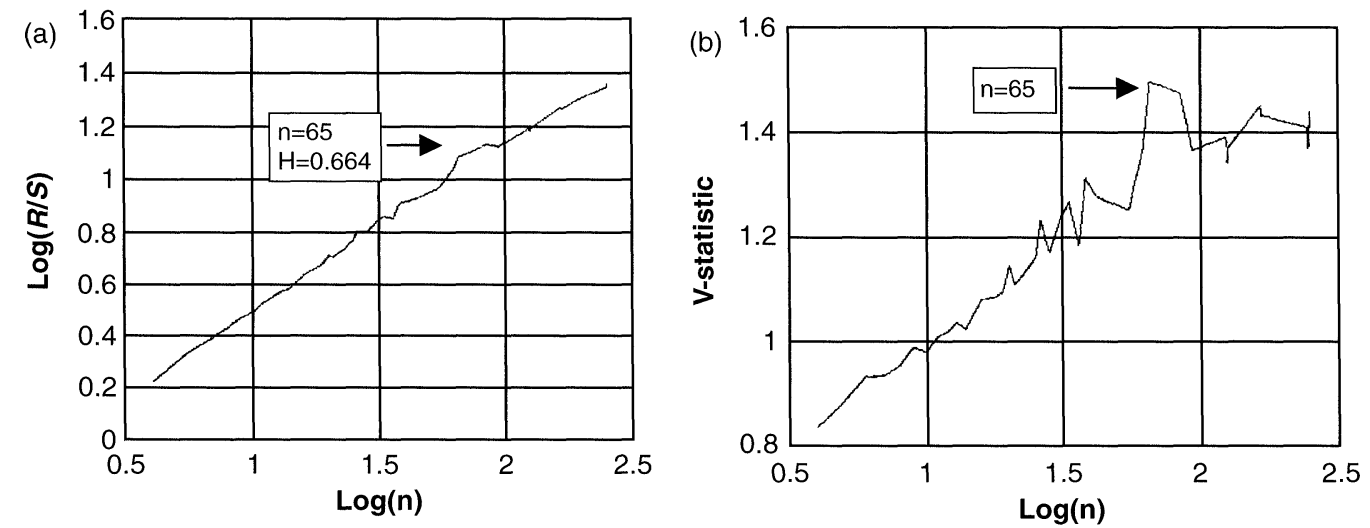

FIGURE $7 \quad R / S$ analysis of the DM/GRD five-day returns (a) Hurst exponent estimation through the $R / S$ statistic plot (b) Cycle-length estimation through the V-statistic plot.

\section{DM/GRD - Twenty day returns}
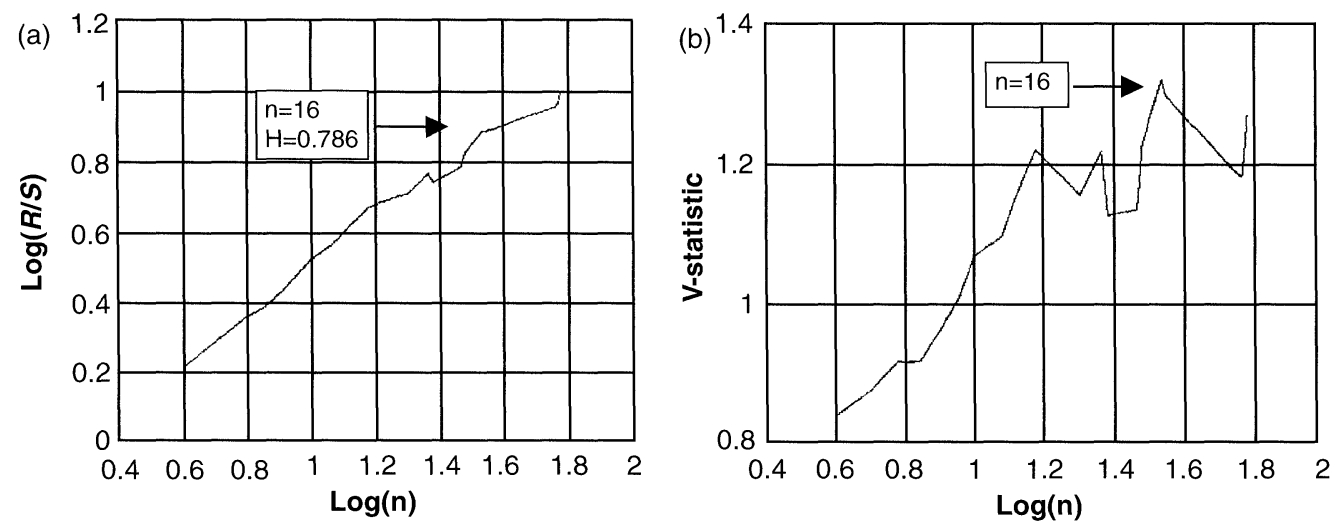

FIGURE $8 \quad R / S$ analysis of the DM/GRD twenty-day returns (a) Hurst exponent estimation through the $R / S$ statistic plot (b) Cycle-length estimation through the V-statistic plot.

TABLE XI Hurst estimates and test of significance against the iid random alternative of the five-day and 20-day DM/GRD returns

\begin{tabular}{lcccc}
\hline & \multicolumn{2}{c}{ 5-day series } & \multicolumn{2}{c}{ 20-day series } \\
\hline A. $R /$ S analysis results & $4<\mathrm{n}<65$ & $65<\mathrm{n}<252$ & $4<\mathrm{n}<16$ & $16<\mathrm{n}<60$ \\
Hurst (H) exponent & 0.664 & 0.574 & 0.786 & 0.565 \\
B. Bootstrapping results & $4<\mathrm{n}<65$ & $65<\mathrm{n}<252$ & $4<\mathrm{n}<16$ & $16<\mathrm{n}<60$ \\
B1. $H_{0}: H>H_{\mathrm{R}}($ iid random alternative) & & & & \\
Mean $H_{\mathrm{R}}$ value & 0.587 & 0.519 & 0.633 & 0.549 \\
Significance level & $0.3 \%$ & $28.7 \%$ & $0.2 \%$ & $43.3 \%$ \\
\hline
\end{tabular}

the $H$ exponents corresponding to them are highly significant at $1 \%$ significance level as Table XI presents. In addition, as in the case of one-day changes, in both the above cases the $\log / \log$ plot crosses over to a random walk since the $H$ exponents corresponding to the data length after the cycle are not significantly different than those from bootstrapped random shuffles. 


\section{The FF/GRD Exchange Rate}

The statistical properties of the series presented in Table XII, as well as, the time series plot in Fig. 9, show a close resemblance to the DM/GRD series.

Unlike the DM series no pre-filtering was necessary due to the lack of any significant autocorrelation. However, when $R / S$ analysis was applied, the same 330-trading day cycle appears (see Fig. 10(a) and (b)) with a slightly lower $H$ estimate of 0.610 , significant at $1 \%$ significance level, as bootstrapping results in Table XIII show. After the cycle $(330<n<1320)$, the series becomes a random walk, as in the DM case.
The modified $R / S$ statistic's results are more intriguing in this case. As Table XIV shows, $V_{q}$ values are lower and marginally significant for low $q$ values when the asymptotic critical values are used. Yet, significance is increased (long-term dependence is not rejected) when the bootstrapped critical values are considered.

The final step is to distinguish between the fractal noise and noisy chaos alternatives. Figures 11(a), (b) and 12(a), (b) show that 5-day and 20-day FF changes exhibit clear cycles of 68 and 15 observations respectively, both approximately equivalent to the 330 one-day cycle.

TABLE XII Statistical description of the FF/GRD daily return series

\begin{tabular}{lclr}
\hline Sample size & 2659 & Range & 0.080023 \\
Average & 0.00021 & Lower quartile & -0.00023 \\
Median & 0.000137 & Upper quartile & 0.00056 \\
Variance & 0.0000026 & Skewness & 27.41 \\
Standard deviation & 0.001617 & Standardized skewness & 577.06 \\
Minimum & -0.01241 & Kurtosis & 1140.01 \\
Maximum & 0.067613 & Standardized kurtosis & 11999.47 \\
\hline
\end{tabular}

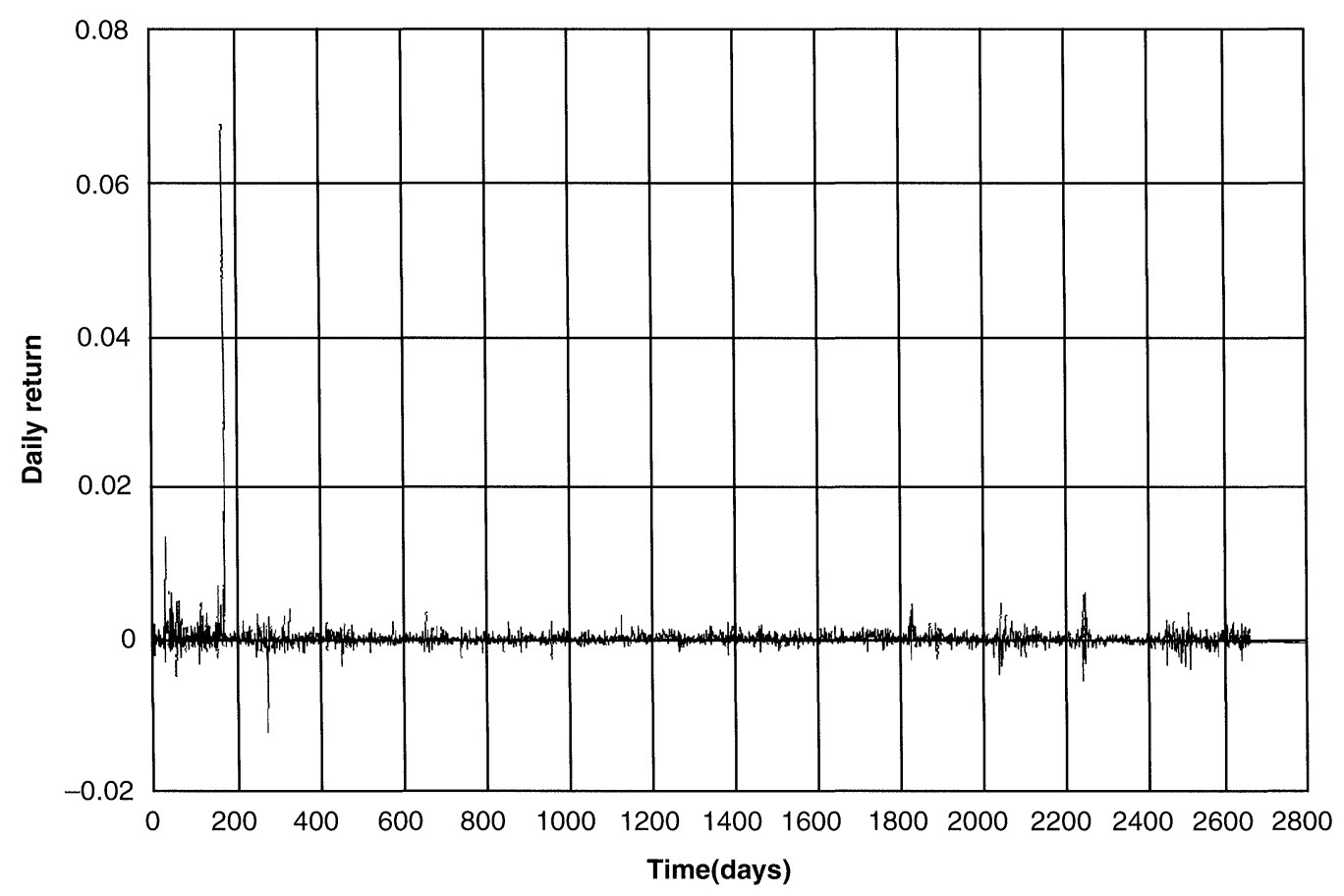

FIGURE 9 Time series plot of the daily returns for the FF/GRD exchange-rate. 
FF/GRD - One day returns
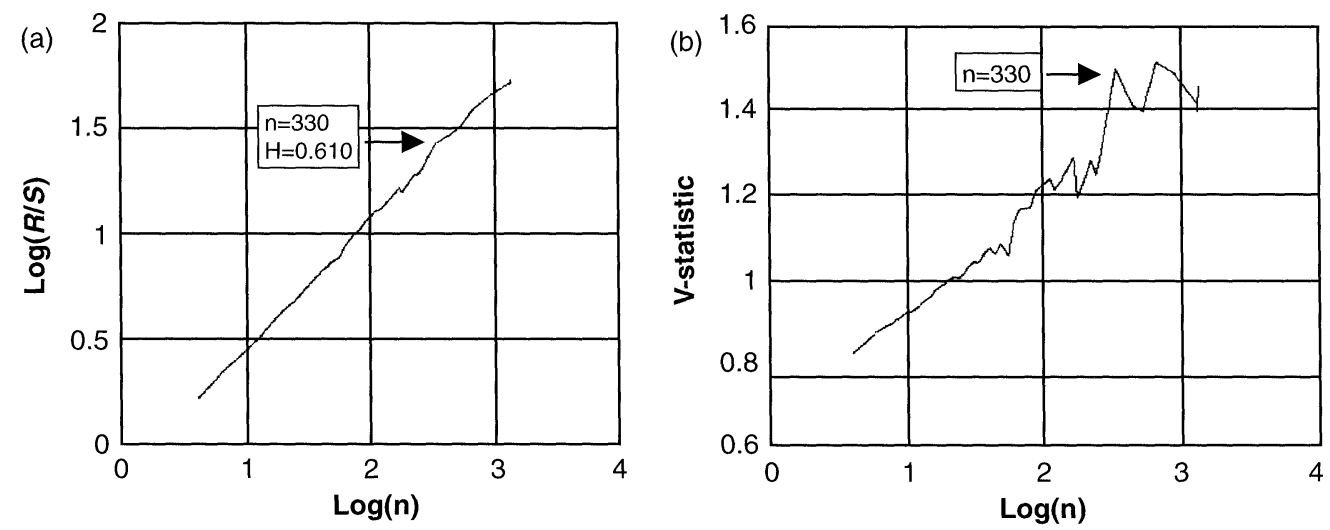

FIGURE $10 \quad R / S$ analysis of the FF/GRD daily returns (a) Hurst exponent estimation through the $R / S$ statistic plot (b) Cyclelength estimation through the V-statistic plot.

TABLE XIII Hurst estimates and test of significance against two random alternatives of the daily FF/GRD returns

\begin{tabular}{lcc}
\hline A. $R / S$ analysis results & $\begin{array}{c}4<\mathrm{n}<330 \\
\text { (Cycle length) } \\
\text { Hurst }(\mathrm{H}) \text { exponent }\end{array}$ & $\begin{array}{c}330<\mathrm{n}<1320 \\
\text { B. Bootstrapping results }\end{array}$ \\
$\begin{array}{l}4<\mathrm{n}<330 \\
\text { (Cycle length) }\end{array}$ & $\begin{array}{c}330<\mathrm{n}<1320 \\
\text { (Remaining data length) }\end{array}$ \\
B1. $H_{0}: H>H_{\mathrm{G}}$ (Gaussian random alternative) & & 0.518 \\
Mean $H_{\mathrm{G}}$ value & 0.562 & 0.503 \\
Significance level & $1.5 \%$ & $41.6 \%$ \\
B2. $H_{0}: H>H_{\mathrm{R}}$ (iid random alternative) & & \\
Mean $H_{\mathrm{R}}$ value & 0.555 & 0.500 \\
Significance level & $0.6 \%$ & $40.0 \%$ \\
\hline
\end{tabular}

TABLE XIV The modified $R / S$ statistic of the daily FF/GRD returns and bootstrapped critical values of the test statistic

\begin{tabular}{lcccc}
\hline$q$ & $\begin{array}{c}\text { Andrew's } \\
\text { q }\end{array}$ & $\begin{array}{c}\mathrm{N}^{1 / 4} \\
7\end{array}$ & $\begin{array}{c}\mathrm{N}^{1 / 3} \\
14\end{array}$ & $\begin{array}{c}\mathrm{N}^{1 / 2} \\
51\end{array}$ \\
\hline$V_{q}$-statistic & 1.662 & 1.62 & 1.53 & 1.36 \\
Level of significance (asymptotic values) & $10 \%$ & $10 \%$ & $>10 \%$ & $>10 \%$ \\
Level of significance (bootstrapped values) & $2.5 \%$ & $5 \%$ & $10 \%$ & $>10 \%$ \\
Bootstrapped critical values & & & & \\
Significance level & & & & \\
$1.0 \%$ & 1.779 & 1.788 & 1.701 & 1.695 \\
$2.5 \%$ & 1.653 & 1.644 & 1.639 & 1.584 \\
$5.0 \%$ & 1.570 & 1.568 & 1.559 & 1.519 \\
$10.0 \%$ & 1.462 & 1.457 & 1.445 & 1.410 \\
\hline
\end{tabular}

The $H$ exponents corresponding the above cycles shown in Table $\mathrm{XV}$, are increasing with longer sampling intervals and highly significant at $1 \%$ significance level. Hence, as in the case of the DM changes, a noisy chaos explanation seems possible.

\section{CONCLUSIONS}

The behavior of four major exchange rate currencies, namely the USD, DM, FF and BP, against Greek Drachma was examined to reveal the 
FF/GRD - Five day returns
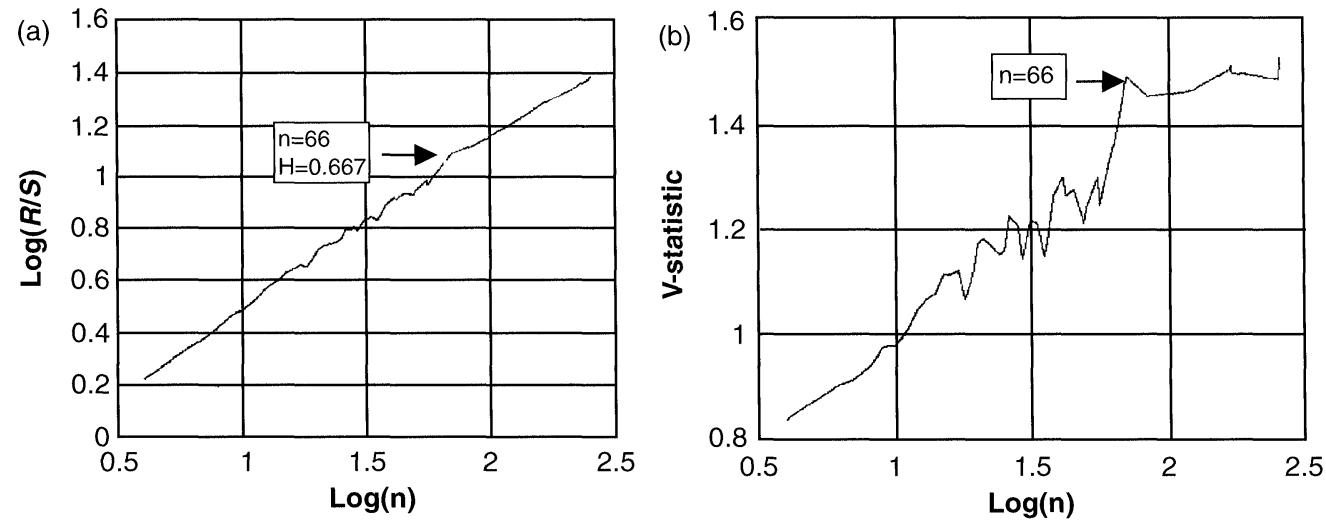

FIGURE $11 R / S$ analysis of the FF/GRD five-day returns (a) Hurst exponent estimation through the $R / S$ statistic plot (b) Cycle-length estimation through the V-statistic plot.

FF/GRD - Twenty-day returns
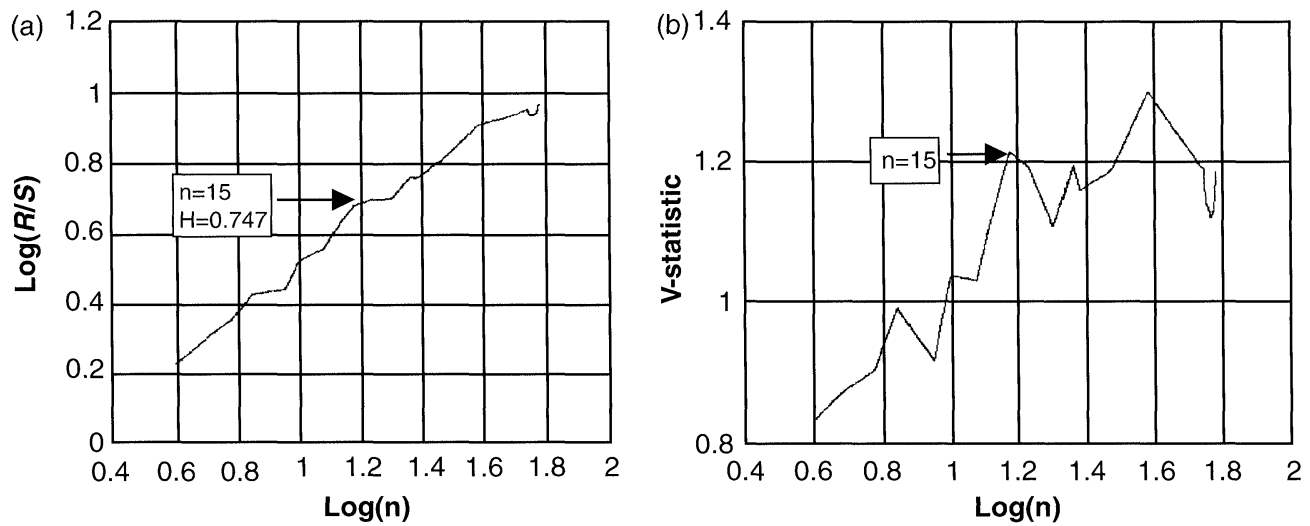

FIGURE $12 R / S$ analysis of the FF/GRD twenty-day returns (a) Hurst exponent estimation through the $R / S$ statistic plot (b) Cycle-length estimation through the V-statistic plot.

TABLE XV Hurst estimates and test of significance against the iid random alternative of the five-day and 20-day FF/GRD returns

\begin{tabular}{lcccc}
\hline & \multicolumn{2}{c}{ 5-day series } & \multicolumn{2}{c}{ 20-day series } \\
\hline A. $R /$ S analysis results & $4<\mathrm{n}<66$ & $66<\mathrm{n}<252$ & $4<\mathrm{n}<15$ & $15<\mathrm{n}<60$ \\
Hurst (H) exponent & 0.667 & 0.553 & 0.747 & 0.507 \\
B. Bootstrapping results & $4<\mathrm{n}<66$ & $66<\mathrm{n}<252$ & $4<\mathrm{n}<15$ & $15<\mathrm{n}<60$ \\
B1. $H_{0}: H>H_{\mathrm{R}}($ iid random alternative) & & & & \\
Mean $H_{\mathrm{R}}$ value & 0.587 & 0.510 & 0.626 & 0.553 \\
Significance level & $0.6 \%$ & $37 \%$ & $0.1 \%$ & $65 \%$ \\
\hline
\end{tabular}

possible existence of long-term dependence. Multiple tests were employed on the time series samples of these currencies and results were compared.

The basic statistical properties showed nonnormal skewness and leptokurtosis for the USD and BP, while the DM and FF statistical distribution exhibited considerably larger skewness and leptokurtic values.

The application of Rescaled Range and $V$ statistics analysis on daily returns revealed evidence 
of cyclical patterns. These results were then tested with bootstrapped methods and the modified $R / S$ test, through Monte Carlo simulations. We found that the alleged cycles for the USD and the BP were just statistical artifacts and not true cycles. On the other hand, our simulation findings confirmed that the cycles of approximately 330 days in length discerned for the DM and FF series were clearly true. Our results were highly significant compared to both the Gaussian and iid random nulls.

Finally we investigated whether these cycles found in DM and FF series were a result of noisy chaos or a fractal noise product. The analysis was repeated for 5-day and 20-day returns. The new series exhibited clear cycles independent of the sample size and equivalent to the 330 one-day cycle. This results in favor of a noisy chaos explanation over fractional Brownian motion.

An interesting thing to notice is the remarkable similarity observed in the behavior of the series in pairs. Both USD and BP on one hand have almost the same statistical characteristics and Hurst exponents, no apparent structure and the cycles found were statistical artifacts. DM on the other hand shows a very close resemblance to the FF, in both the statistical structure and the true cyclical patterns discerned, of 330 days length.

These results seek for further economic interpretation. In the Greek economy exchange rate policy has a very important role and is been exercised by setting a target depreciation rate at the beginning of each planning period in year-end terms vs. the ECU. For the period under review, this policy has provided for a dramatic devaluation of drachma in 1985, shown by sharp peaks in all the time series plots. Since then depreciation rates have declined and at the end of the testing period annual depreciation was less than $4 \%$ acting as an antiinflationary instrument in anticipation of the drachma participation to the Exchange Rates Mechanism (ERM). The point is that the policy that has been followed provides for increased degree of discipline for the drachma fluctuations vs. all ECU participant currencies that include DM, FF and BP. Hence, a more random behavior of the GRD/USD exchange rate could be justified, as well as, a more structured one for DM and FF. In this context the behavior of the $\mathrm{BP}$ is intriguing. A possible explanation in this case could lie with the additional discipline imposed on the system due to the participation of DM and FF to ERM. The $\mathrm{BP}$ on the contrary has participated the ERM only for a short period until its membership suspension on the 16th of September 1992, a period too short to provide for a disciplined behavior within the sample period chosen.

The conclusion, therefore, is that the results obtained are justifiable and the explanation lies with the degree of discipline of behavior that characterizes the fluctuations of each of the currencies involved vs. GRD.

Finally, we should remark that the DM and FF time series should be further investigated as to show whether their behavior conforms with a chaotic one. Since a noisy chaos explanation has been supported by this research, tools from the nonlinear dynamics field should be used to verify the existence of and to reveal chaotic characteristics in these two series.

\section{References}

[1] Andrews, D.: Heteroscedasticity and autocorrelation consistent covariance matrix estimation. Econometrica $\mathbf{5 9}$, 817-858 (1991).

[2] Anis, A.A. and Lloyd, E.H.: The expected value of the adjusted rescaled Hurst range of independent normal summands. Biometrica 63, 155-164 (1976).

[3] Aydogan, K. and Booth, G.: Are there long cycles in common stock returns? Southern Economic Journal 55, 141-149 (1988).

[4] Booth, G.G. and Koveos, P.E.: Employment fluctuations: an $R / S$ analysis. Journal of Regional Science 23, 19-31 (1983).

[5] Booth, G.G., Kaen, F.R. and Koveos, P.E.: Currency interdependence in foreign exchange markets. The Financial Review 15, 36-44 (1980).

[6] Booth, G.G., Kaen, F.R. and Koveos, P.E.: Persistent dependence in gold prices. Journal of Financial Research $\mathbf{5}$, 85-93 (1982a).

[7] Booth, G.G., Kaen, F.R. and Koveos, P.E.: R/S analysis of foreign exchange rates under two international monetary regimes. Journal of Monetary Economics 10, 407-415 (1982b).

[8] Brock, W.A. and DeLima, J.F.: Nonlinear time series, complexity theory and finance. The Handbook of Statistics, G.S. Maddala and C.R. Rao, Vol. 14, Amsterdam: North Holland (1995).

[9] Campbell, J. and Mankiw, G.: Are output fluctuations transitory? Quarterly Journal of Economics 102, 857-880 (1987). 
[10] Cheung, Y. and Lai, K: Do gold markets have longmemory? Journal of Business and Economic Statistics 11, 93-101 (1993).

[11] Cheung, Y., Lai, K. and Lai, M.: Are there long cycles in foreign stock returns? Journal of International Financial Markets, Institutions and Money 3, 33-47 (1993).

[12] Crato, N.: Some international evidence regarding the stochastic memory of stock returns. Applied Financial Economics 4, 33-39 (1994).

[13] Davies, R. and Harte, D.: Tests for Hurst effect. Biometrica 74, 95-102 (1987).

[14] Efron, B.: Bootstrap methods: Another look at the jackknife. The Annals of Statistics 7, 1-26 (1979).

[15] Fama, E. and French, K.: Permanent and temporary components of stock prices. Journal of Political Economy 96, 246-273 (1988).

[16] Gewke, J. and Feige, E.: Some joint tests of the efficiency of markets for forward exchange. Review of Economics and Statistics 61, 334-341(1979).

[17] Glassman, D.: The efficiency of foreign exchange futures markets in turbulent and non-turbulent periods. The Journal of Futures Markets 7, 245-267 (1987).

[18] Granger, C. and Joyeux, R.: An introduction to long-range time series models and fractional differencing. Journal of Time Series Analysis 1, 15-30 (1980).

[19] Greene, M. and Fielitz, B.: Long-term dependence in common stock returns. Journal of Financial Economics 4, 339-349 (1977).

[20] Haubrich, J.G. and Lo, A.W.: The sources and nature of long-term memory in business cycle, Working Paper, \# 589, Rodney L. White Center for Financial research, The Wharton School, University of Pennsylvania, Philadelphia (1989).

[21] Helms, B., Kaen, F. and Rosenman, R.: Memory in commodity futures contracts. The Journal of Futures Markets 4, 559-567 (1984).

[22] Hiemstra, G. and Jones, J.D.: Another look at long memory in common stock returns, Discussion Paper, University of Strathclyde (1995).

[23] Hosking, J.: Fractional differencing. Biometrika 68, 165176 (1981).

[24] Hsieh, D.A.: The statistical properties of daily foreign exchange rates: 1974-1983. Journal of International Economics 24, 129-145 (1988).

[25] Hsieh, D.A.: Modelling heteroscedasticity in daily foreign exchange rates. Journal of Business and Economic Statistics 7, 307-317 (1989a).

[26] Hsieh, D.A.: Testing for nonlinear dependence in daily foreign exchange rates. Journal of Business 62, 339-368 (1989b).

[27] Hurst, H.E.: Long-term storage capacity of reservoirs. Transactions of the American Society of Civil Engineers, 116 (1951).

[28] Hurst, H.E.: Methods of using long-term storage in reservoirs. Proceedings of the Institute of Civil Engineers 1, 519-543 (1956).

[29] Kao, G.W. and Ma, C.K.: Memories, heteroscedasticity and price limit in currency futures markets. The Journal of Futures Markets 12, 679-692 (1992).
[30] Lo, A.W.: Long term memory in stock market prices. Econometrica 59, 1279-1313 (1991).

[31] Lo, A. and Mackinlay, A.C.: Stock market prices do not follow random walks: evidence from a simple specification test. Review of Financial Studies 1, 41-66 (1988).

[32] Loteran, M. and Phillips, P.: Testing the covariance stationarity of heavy tailed time series: An overview of the theory with applications to several financial data sets. Journal of Empirical Finance 1, 211-248 (1994).

[33] Mandelbrot, B.: Statistical methodology for non-periodic cycles: from the covariance to $R / S$ analysis. Annals of Economic and Social Measurement 1 (1972).

[34] Mandelbrot, B. and Wallis, J.: Computer experiments with fractional gaussian noises. Part 1, averages and variances. Water Resourses Research 5 (1969a).

[35] Mandelbrot, B. and Wallis, J.: Computer experiments with fractional gaussian noises. Part 2, rescaled ranges and spectra. Water Resources Research 5 (1969b).

[36] Mandelbrot, B. and Wallis, J.: Computer experiments with fractional gaussian noises. Part 3, mathematical appendix. Water Resources Research 5 (1969c).

[37] Mandelbrot, B. and Wallis, J.: Robustness of the rescaled range $R / S$ in the measurement of non-cyclic long run statistical dependence. Water Resources Research 5 (1969d).

[38] Messe, R.A. and Singleton, K.J.: On the unit roots and the empirical modeling of exchange rates. Journal of Finance 37, 1029-1034 (1982).

[39] Mills, T.: How robust is the finding that innovations to U.K. output are persistent? Scottish Journal of Political Economy 39, 154-166 (1992).

[40] Mills, T.: Is there long-term memory in UK stock returns? Applied Financial Economics 3, 303-306 (1993).

[41] Milonas, N., Koveos, P. and Booth, G.: Memory in commodity futures contracts: a comment. The Journal of Futures Markets 5, 113-114 (1985).

[42] Noreen, E.W.: Computer Intensive Methods for Testing Hypotheses, New York: John Wiley \& Sons, 1989.

[43] Peters, E.: Fractal structure in the capital markets. Financial Analysts Journal, July/August (1989).

[44] Peters, E.: Chaos and Order in the Capital Markets: A New View of Cycles, Prices and Market Volatility. New York: John Wiley \& Sons, 1991a.

[45] Peters, E.: A chaotic attractor for the S\&P 500. Financial Analysts Journal, March/April (1991b).

[46] Peters, E.: $R / S$ analysis using logarithmic returns: a technical note. Financial Analysts Journal, November/ December (1992).

[47] Peters, E.: Fractal Market Analysis: Applying Chaos Theory to Investment and Economics. New York: John Wiley \& Sons, 1994.

[48] Phillips, P.: Time series regression with a unit root. Econometrics 55, 277--301 (1987).

[49] Poterba, J. and Summers, L.: Mean reversion in stock returns: evidence and implications. Journal of Financial Economics 22, 27-60 (1988).

[50] Rosenblatt, M.: A central limit theorem and a strong mixing condition. Proceedings of the National Academy of Sciences 42, 43-47 (1956). 


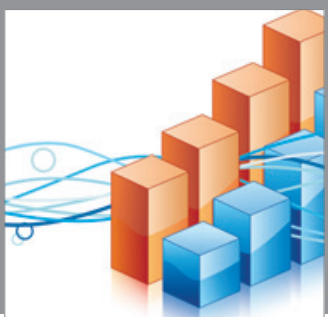

Advances in

Operations Research

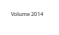

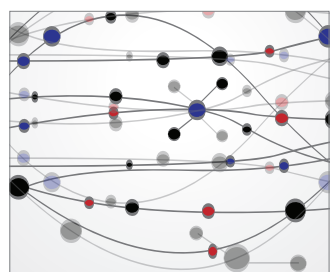

\section{The Scientific} World Journal
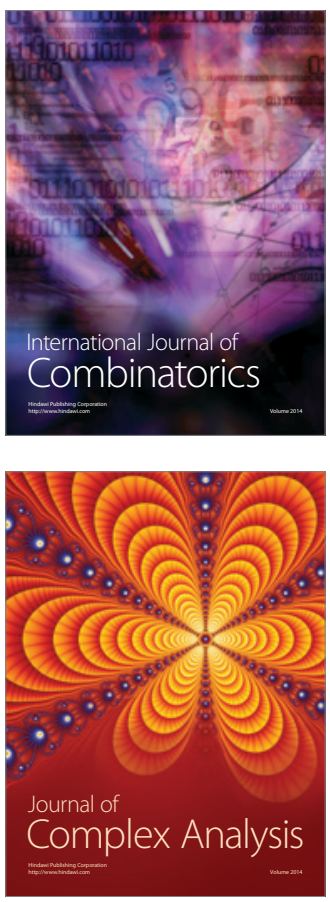

International Journal of

Mathematics and

Mathematical

Sciences
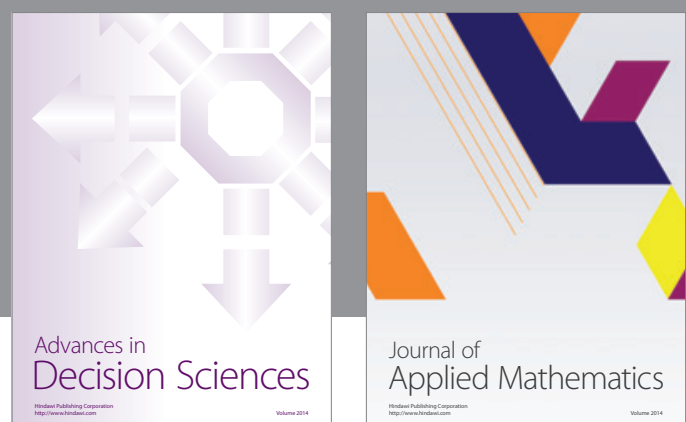

Journal of

Applied Mathematics
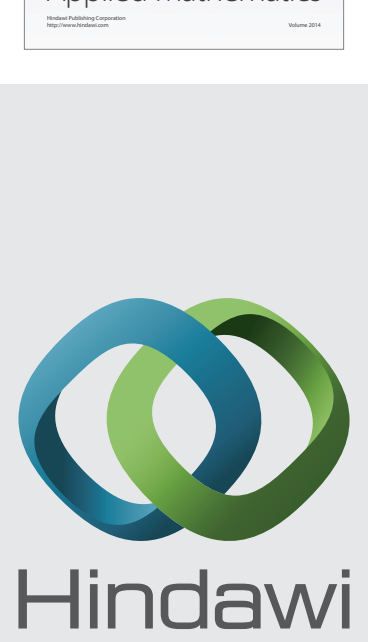

Submit your manuscripts at http://www.hindawi.com
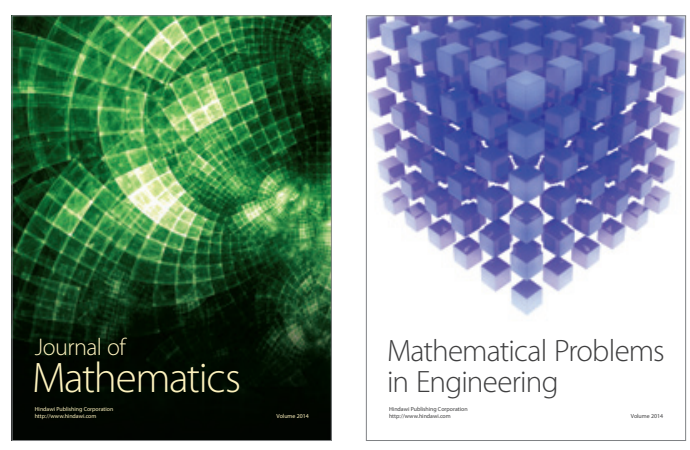

Mathematical Problems in Engineering
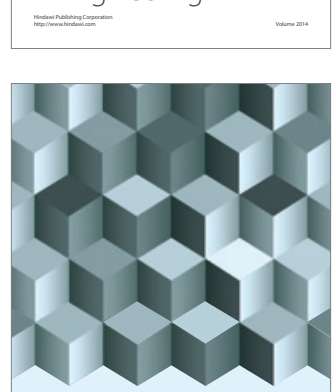

Journal of

Function Spaces
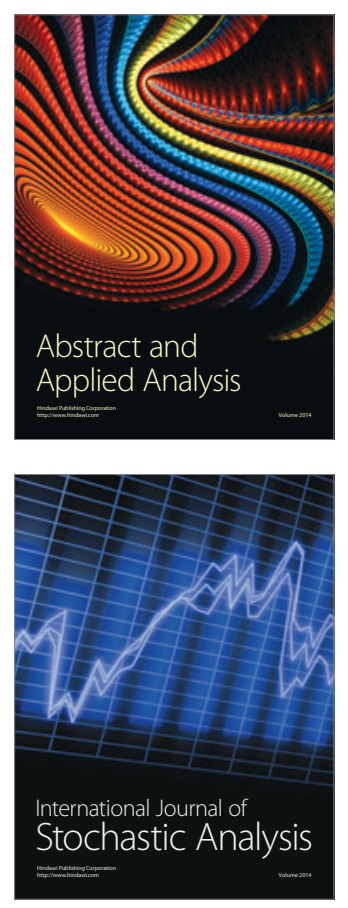

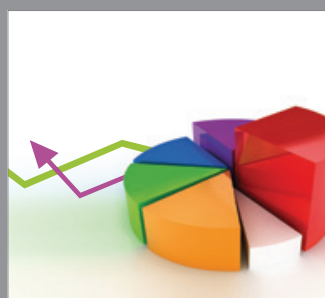

ournal of

Probability and Statistics

Promensencen
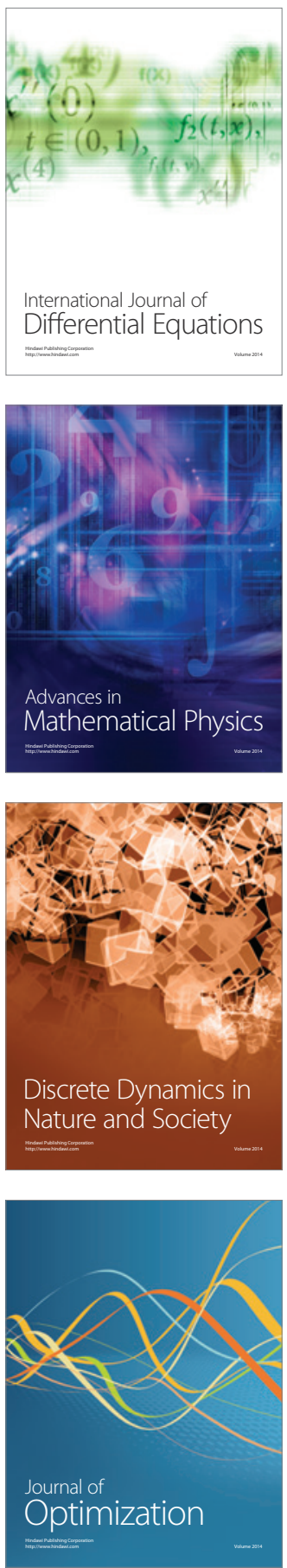